\title{
Proper evaluation of chemical cross-linking- based spatial restraints improves the precision of modeling homo-oligomeric protein complexes
}

\author{
Aljaž Gaber, Gregor Gunčar and Miha Pavšič (1)
}

\begin{abstract}
Background: The function of oligomeric proteins is inherently linked to their quaternary structure. In the absence of high-resolution data, low-resolution information in the form of spatial restraints can significantly contribute to the precision and accuracy of structural models obtained using computational approaches. To obtain such restraints, chemical cross-linking coupled with mass spectrometry (XL-MS) is commonly used. However, the use of $\mathrm{XL}-\mathrm{MS}$ in the modeling of protein complexes comprised of identical subunits (homo-oligomers) is often hindered by the inherent ambiguity of intra- and inter-subunit connection assignment.

Results: We present a comprehensive evaluation of (1) different methods for inter-residue distance calculations, and (2) different approaches for the scoring of spatial restraints. Our results show that using Solvent Accessible Surface distances (SASDs) instead of Euclidean distances (EUCs) greatly reduces the assignation ambiguity and delivers better modeling precision. Furthermore, ambiguous connections should be considered as inter-subunit only when the intra-subunit alternative exceeds the distance threshold. Modeling performance can also be improved if symmetry, characteristic for most homo-oligomers, is explicitly defined in the scoring function.
\end{abstract}

Conclusions: Our findings provide guidelines for proper evaluation of chemical cross-linking-based spatial restraints in modeling homo-oligomeric protein complexes, which could facilitate structural characterization of this important group of proteins.

Keywords: Chemical cross-linking, Homo-oligomers, Cross-link assignation ambiguity, Solvent accessible surface distances, Modeling

\section{Background}

To understand the mechanisms of how proteins drive and regulate biological processes, detailed knowledge of their structure is fundamental. Obtaining structural information is a long and complicated process, even more so when the object of investigation is a complex of multiple proteins. Despite advances in methods for high-resolution structure determination, structural characterization of protein complexes often relies on computational algorithms for protein-

\footnotetext{
* Correspondence: miha.pavsic@fkkt.uni-lj.si

Department of Chemistry and Biochemistry, Faculty of Chemistry and

Chemical Technology, University of Ljubljana, Večna pot 113, 1000 Ljubljana, SI, Slovenia
}

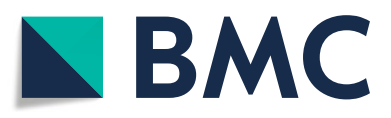

(๑) The Author(s). 2019 Open Access This article is distributed under the terms of the Creative Commons Attribution 4.0 International License (http://creativecommons.org/licenses/by/4.0/), which permits unrestricted use, distribution, and reproduction in any medium, provided you give appropriate credit to the original author(s) and the source, provide a link to the Creative Commons license, and indicate if changes were made. The Creative Commons Public Domain Dedication waiver (http://creativecommons.org/publicdomain/zero/1.0/) applies to the data made available in this article, unless otherwise stated.

protein docking that are guided by spatial restraints obtained from experimental data [1]. Such information, albeit being of low resolution, substantially improves docking accuracy and precision [2]. This approach is also often employed to model the quaternary structure of protein complexes comprised of multiple identical subunits (homo-oligomers), and can be successful when the high-resolution structure of a subunit is available. This is important because $30-50 \%$ of all proteins can self-associate to form such multi-subunit complexes [3]. Here, spatial restraint-driven docking not only helps in the determination of the quaternary structure of protein complexes but can also provide valuable insight into the mechanism of protein self-association $[4,5]$. 
Among the most frequently used spatial restraints are those acquired from chemical cross-linking, coupled with mass spectrometry (XL-MS) [6-8]. Cross-linker, a bi-reactive chemical component, connects specific amino acid residues located at an appropriate distance in the $3 \mathrm{D}$ structure of the protein complex. The length of the cross-linker defines the maximal distance between the reactive ends of the cross-linked residues. This length, combined with the length of the side chains of connected residues and increased for a factor accounting for the protein's backbone flexibility, is used to determine the upper limit of the distance between the $C_{\alpha}$ atoms $\left(C_{\alpha}-C_{\alpha}\right.$ distance) of the cross-linked residues [9]. In the distance restraint-guided docking, multiple models of complexes are generated and used to calculate inter-residue distances of experimentally identified cross-links. To confirm or reject individual models, distances calculated from these models are compared with the theoretical upper limit distances; if a model-derived distance fits the theoretically possible distance limit, the model could be considered as probable.

However, modeling of homo-oligomeric complexes based on XL-MS spatial restraints involves a challenge that is absent from heterogenous complexes, namely all interacting subunits have the same amino acid sequence. As sequences of the connected peptides are derived from their mass spectra, and identification of the cross-linked residues is in turn sequence-based, intra- and inter-subunit connections are therefore inherently ambiguous. Here, each identified connection can be either a result of intra- or inter-subunit connection or both. In homo-dimers, this problem can be circumvented by labeling a single subunit with heavy isotopes during protein expression [10-13], and comparative mass spectrometry approaches have been developed to distinguish between intra- and inter-subunit connections based on the abundance of individual cross-links [14-16]. However, both approaches have their limitations and do not apply to all investigations.

To better understand the ambiguity problem and its possible solutions, we investigated how inter-residue distance in combination with different computational approaches can help to resolve the cross-link assignation ambiguity. Two aspects were considered: (1) methods for inter-residue distance calculations, and (2) scoring approaches for proper evaluation of ambiguous crosslinks as spatial restraints. We performed our investigation by analyzing available high-resolution structures of homo-oligomers. Here we calculated their inter-residue distances using two different methods and compared the precision of protein-protein docking predictions aimed to recreate initial structures by using simulated crosslinking data together with different scoring approaches. For the first part, we analyzed whether using Solvent
Accessible Surface Distances (SASDs) better discriminates between intra- and inter-subunit connections than most commonly used Euclidean distances (EUCs). While SASDs account for residue solvent accessibility and also for space occupied by proteins, EUCs completely disregard both which can lead to false positive assignations $[8,17,18]$ (Fig. 1). In the second part, we compared the effect of different scoring approaches since currently there is no consensus on how to properly score ambiguous cross-links (or whether to use them as spatial restraints at all). Third, we examined how can symmetry, present in almost all homo-oligomers, be used as additional restraint for modeling to give the best possible outcome and also whether it can be used to resolve the ambiguity. Our results provide basic guidelines for efficient use of XL-MS data in modeling homo-oligomers.

\section{Results}

Solvent accessible surface distances are better at distinguishing between intra- and inter-subunit connections

Calculating SASDs instead of EUCs has been shown to improve the precision of modeling proteins and protein complexes, by more accurately defining which inter-residue distances agree with the experimental data $[8,18,19]$. We wanted to evaluate if calculating SASDs instead of EUCs can also help distinguish between intra- and intersubunit cross-links in homo-oligomers. We calculated non-redundant $\mathrm{C} \alpha-\mathrm{C} \alpha$ SASDs and EUCs between lysine residues in 13,110 high-resolution homo-oligomeric protein complex structures (see Materials and Methods). Connections between individual residues were then assigned, based on the corresponding inter-residue distances: Intra-subunit, Inter-subunit, Ambiguous, if both Intra-subunit and Inter-subunit alternatives were valid, or Non-accessible if the distance was too long or the endpoint residue was not accessible to the cross-linker. We used $33 \AA$ as the upper threshold for the assignation - the distance used as a threshold for two commonly used cross-linkers disuccinimidyl suberate (DSS) and bis(sulfosuccinimidyl) suberate (BS3) [18].

Our results show that using SASDs yields a smaller fraction of Ambiguous assignations (11\%) than using EUCs (23\%) (Fig. 2a). Consequently, the fractions of unambiguous assignations are increased: the fraction of Intra-subunit assignations is increased by $5 \%$ from 67 to $72 \%$ and the fraction of Inter-subunit assignations is increased by $7 \%$ from 10 to $17 \%$. On the other hand, more than half $(53 \%)$ of residue pairs could be assigned neither as Intra- nor as Inter-subunit (Fig. 2a, label Non-acc.). A large fraction of Nonaccessible assignations can be explained by the fact that SASDs are longer than EUCs and thus more likely above than the threshold. The sum of median 


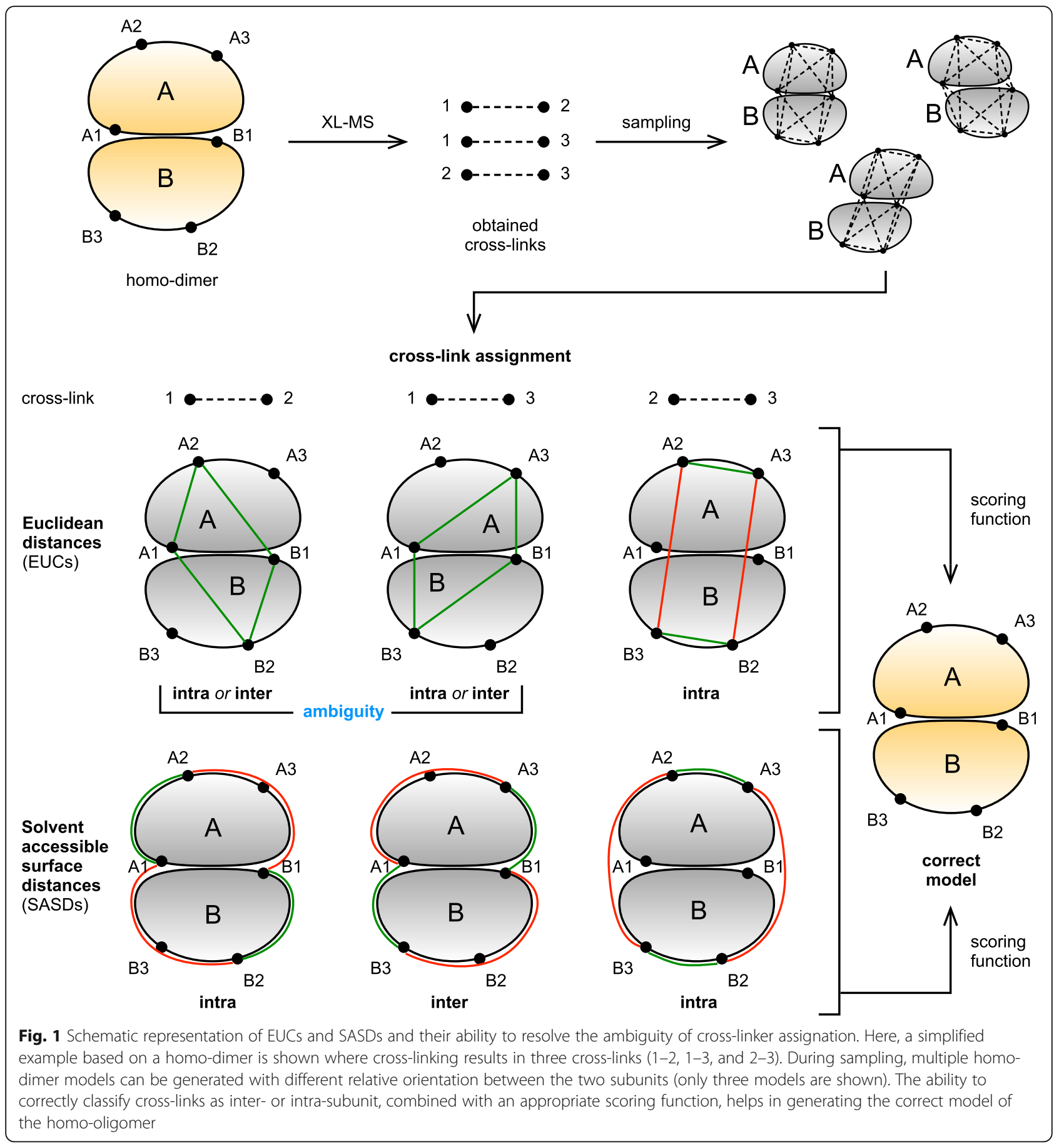

EUC and a median increase of distance when SASDs are calculated for intra-subunit and inter-subunit distances are $33.64 \AA$ and $35.37 \AA$, respectively (Additional file 1 : Figure S1) - that is comparable to our threshold of $33 \AA$.

To test if the rise of the distances that are above the threshold is also the main reason for the reduction of the number of ambiguous assignations with SASD, we investigated if the same effect can be obtained when lowering the threshold to 30,27 and $25 \AA$ when using EUCs. The fraction of Ambiguous assignation did indeed decrease with reducing threshold (Fig. 2b, Additional file 1: Figure S2). While thresholds of 30 and $27 \AA$ still yielded a higher fraction of Ambiguous assignations than using SASDs (17 and 14\%, respectively), the same result of $11 \%$ could be obtained when using a threshold of $25 \AA$. However, reducing threshold did not increase the fraction of 

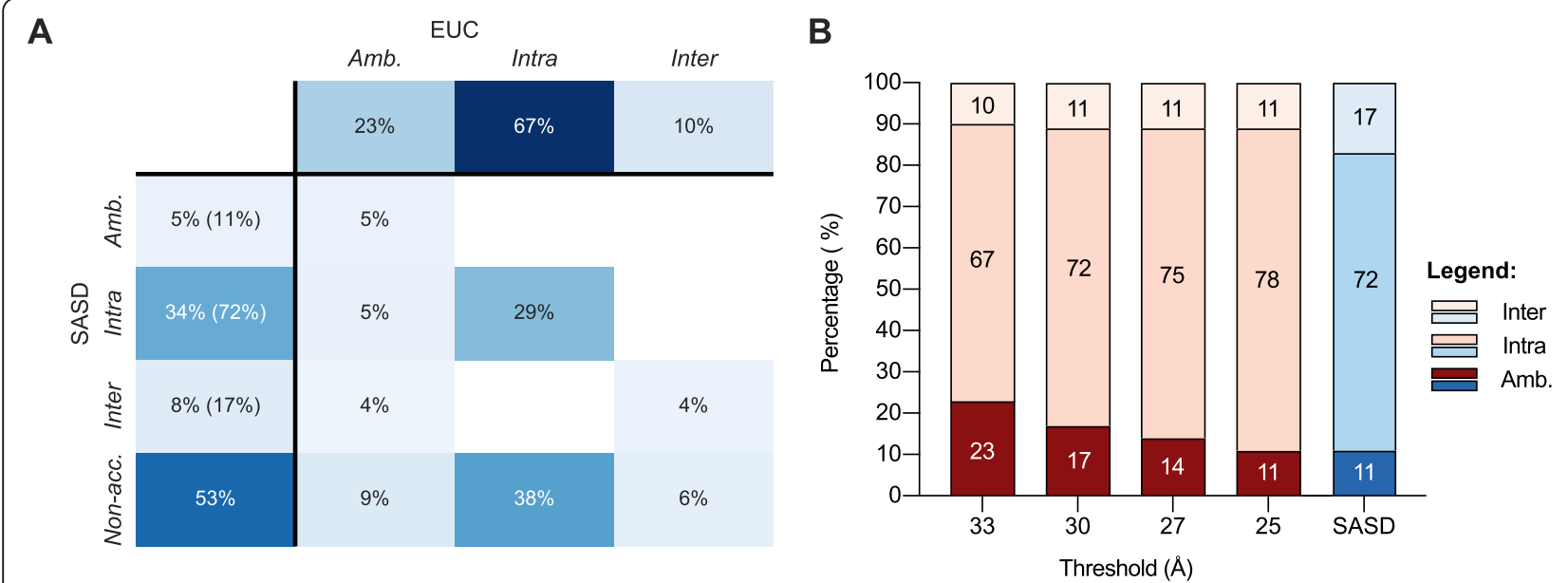

C

D
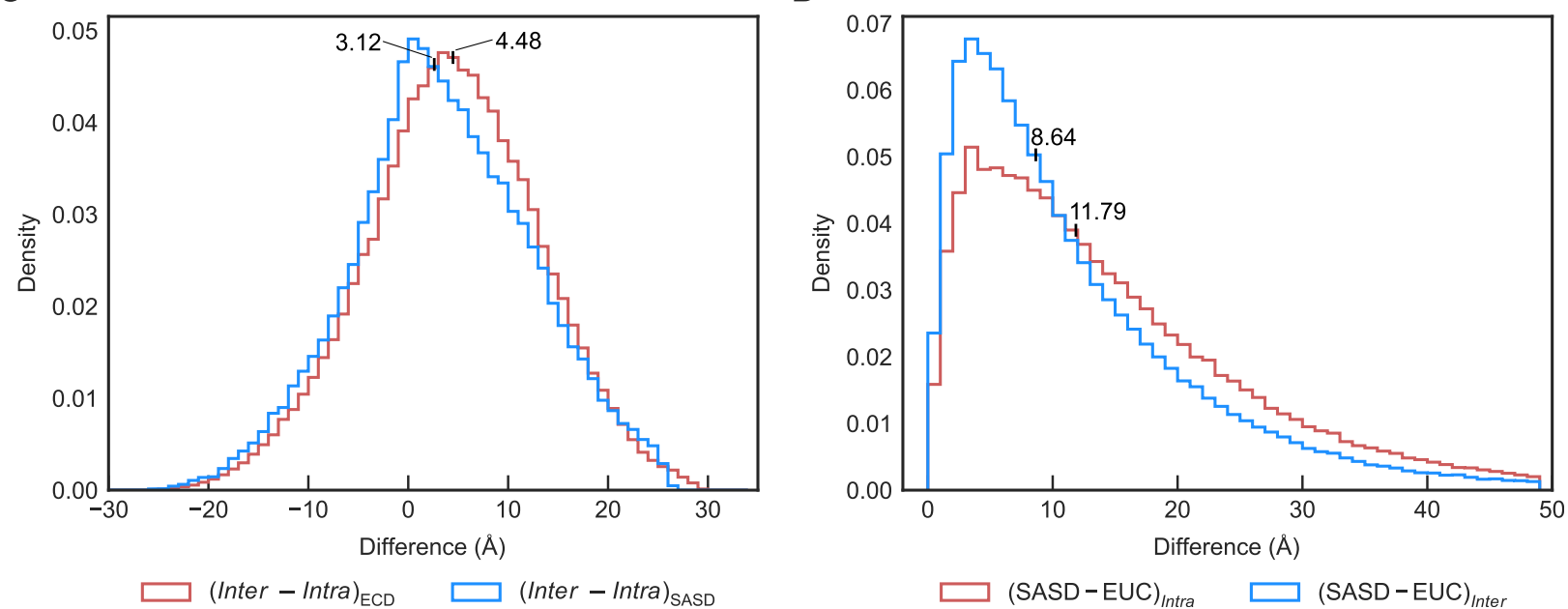

Fig. 2 Comparison of using SASDs over EUCS to reduce the Intra- vs. Inter-subunit ambiguity based on the inter-residue distance. a A heatmap showing a redistribution of assignations when using SASDs over EUCs. Values represent the proportion of individual assignations. The total values obtained with each method are shown in header row and column, while the body of the heatmap shows the proportions of combinations. Values in parentheses represent the relative proportion of assignations obtained using SASDs, excluding Non-accessible. b Comparison of redistribution of assignations between different EUC thresholds (red bars) and using SASDs over EUCs (blue bars). Values inside bar sections represent the relative population of individual assignations. c Distribution of differences between Inter- and Intra-subunit distances. Median values were calculated from kernel density estimates [20]. $\mathbf{d}$ Distribution of differences between calculated SASDs and EUCs. Median values were calculated from kernel density estimates [20]

Inter-subunit assignations as it did in the case of SASDs. Furthermore, the majority of initial ambiguous assignations were assigned as Ambiguous or Intra-subunit, while they were almost equally redistributed between Ambiguous (5\%), Intra-subunit (5\%) and Inter-subunit (4\%) when using SASDs (Fig. 2a). This suggests that using SASDs has a somewhat smaller overall effect on the increase of Inter-subunit distances than it does on the increase of Intra-subunit distances. To confirm this assumption, we compared differences between Inter- and Intra-subunit distances in Ambiguous assignations using both methods. Results show that these differences are normally distributed when calculating
EUCs with a median difference of $4.48 \AA$. However, the distribution is slightly skewed towards smaller differences when using SASDs (inter-subunit distances are less often longer than intra-subunit; Fig. 2c). The smaller overall effect on Inter-subunit distances is also evident if we compare the increase of Intra- and Inter-subunit distance when SASDs are used over EUCs (Fig. 2d). The median difference is $11.79 \AA$ in case of Intra-subunit distances, compared to a median increase of 8.64 $\AA$ in case of Inter-subunit distances.

Our analyses, taken together, clearly show that using SASDs instead of EUCs provides significant improvement in reducing the ambiguity of XL-MS data assignation 
based on the calculated inter-residue distances alone. Additionally, using SASDs results in a higher number of identified Inter-subunit connections, which are used as spatial restraints in the modeling of homo-oligomeric protein complexes.

\section{Considering the Intra-subunit alternative improves the results of modeling of homo-oligomeric protein complexes}

Even though our results show that using SASDs instead of Euclidean distances can reduce the ambiguity of cross-link assignation, some cross-links will remain ambiguous. We investigated the effect of the scoring function to score ambiguous cross-links as Inter- or Intra-subunit on the precision of modeling results.

Ideally, the performance of different scoring approaches would be assessed on a representative dataset of homo-oligomeric protein complexes with different stoichiometries. However, modeling of higher-thandimer order homo-oligomers introduces another level of ambiguity as each identified inter-residue connection also has multiple ambiguous Inter-subunit alternatives. To simplify the comparison, we focused on symmetric homo-dimers only, as they are also by far most predominate oligomeric state within homo-oligomers [3].

We performed our analysis using a representative dataset of 41 homo-dimers. XL-MS data was simulated by extracting lysine residue pairs with inter-residue SASDs below the threshold ( $33 \AA$ ) and used to guide protein-protein docking by assigning each potential cross-link a Matched and Non-accessible cross-link (MNXL) [18] score. MNXL score distinguishes between cross-links that have inter-residue distance below the threshold (matched) and those which don't (non-accessible). We then used different scoring functions to calculate the total inter-subunit MNXL score of a model and compared the highestranking models with the initial structure of homodimer to assess scoring function's precision (for more details see Materials and Methods).

First, we examined if treating ambiguous cross-links as ambiguous instead of designating them as Inter-subunit to obtain the maximal number of spatial restraints, is indeed important. To do so, we created the following scoring functions:

- the first one scored all ambiguous cross-links as Inter-subunit and was oblivious to the existence of Intra-subunit alternative (Oblivious);

- the second one scored Inter-subunit alternative but did not penalize non-accessible Inter-subunit crosslinks, if Intra-subunit alternative was matched (Normal).
Because scoring algorithms usually address cross-links as oriented (i.e., a cross-link between subunits A-B is not the same as a cross-link between subunits B-A, which is otherwise the case in homo-oligomers), two variations of scoring functions were used:

- one that addressed cross-links as oriented and thus evaluated alternatives A-B and B-A separately (oriented) and

- one that considered only the alternative with the highest MNXL score (stringent).

Results of the comparison show that Normal scoring functions have higher precision than their Oblivious counterparts with both methods used for distance calculation (SASD and EUC) (Fig. 3a, Additional file 2: Table S2)). Even tough Oblivious scoring functions use more spatial restraints per model, penalizing non-accessible Inter-subunit connections, when Intra-subunit counterpart is matched, greatly reduces the precision. This demonstrates that Intraand Inter-subunit alternatives should always be considered as a single restraint and not scored individually.

Surprisingly, evaluating Inter-subunit alternatives individually improves the precision of protein-protein docking, as oriented scoring functions have much higher precision than stringent scoring functions (Fig. 3a).

With regards to the method of choice for inter-residue distance calculation, SASDs give better results compared to EUCs (Fig. 3b), although the difference is not statistically significant when using Oblivious-oriented scoring function. This supports our previous findings that SASDs are better at resolving the ambiguity of Intra- vs. Inter-subunit assignation.

\section{Only the cross-links that cannot be matched as Intra-} subunit should be considered as Inter-subunit restraints After establishing that Intra-subunit alternative should be considered when Inter-subunit alternative is non-accessible, we examined in which case a cross-link should be scored as Inter-subunit (with regards to the inter-residue distances) if both alternatives are possible. We considered three options:

- option All scored all cross-links as Inter-subunit, even if the Intra-subunit option was matched and had a higher score;

- option Only best-scored cross-links as Inter-subunit only if the Inter-subunit score was higher than the Intra-subunit;

- option Non-Intra had the strictest criterium and scored cross-links as Inter-subunit only if the Intrasubunit alternative was non-accessible.

These three options were applied to Normal-oriented and Normal-stringent scoring functions introduced above. 


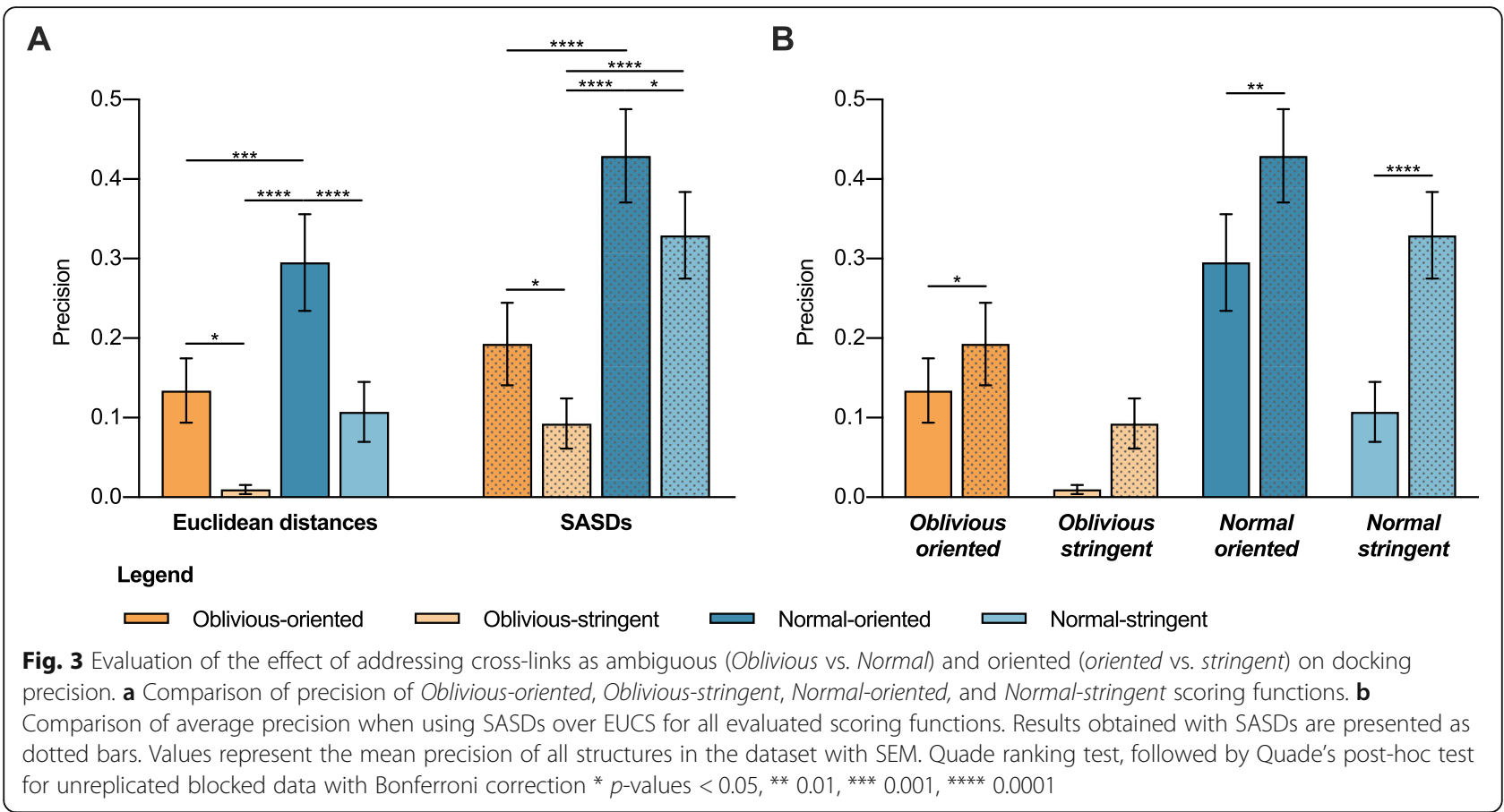

Results suggest that the more conservative scoring function is better. The Non-Intra option gives the highest precision in all analyzed scoring functions, followed by Only best and All, which gives the lowest precision (Fig. 4a). The difference between Non-Intra and All is statistically significant in all cases, except when using
Normal-oriented scoring functions and calculating EUCs. On the other hand, the difference between Only best and All is not statistically significant, even though the average precision of the former is higher in all cases.

In agreement with our previous findings, Normal-oriented always yields better results than Normal-stringent
A

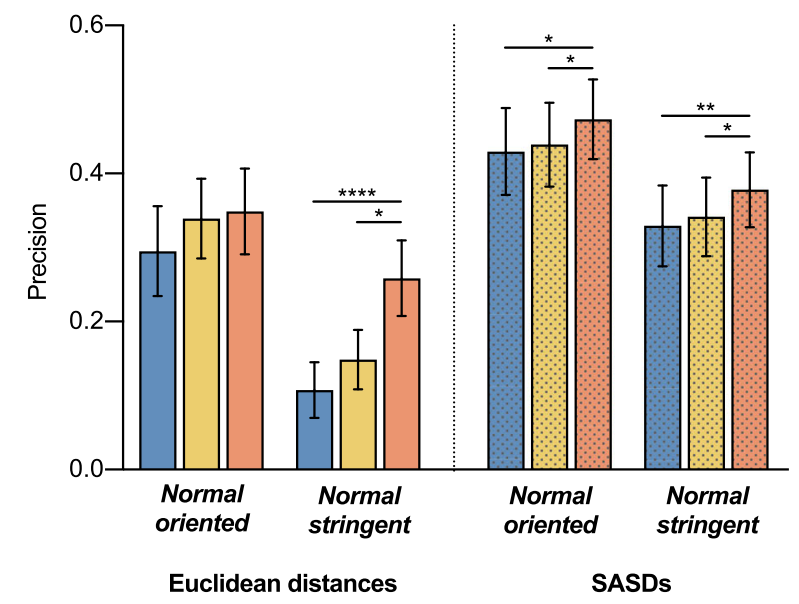

B

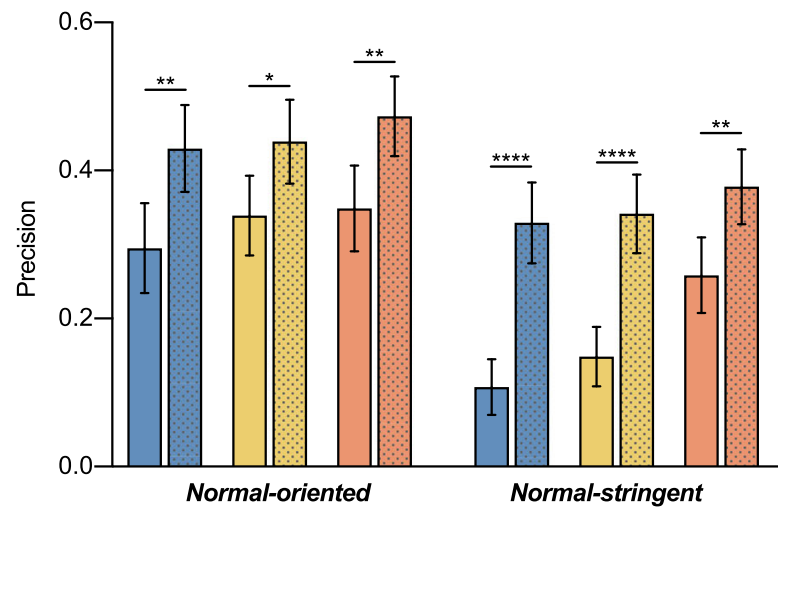

\section{Legend}

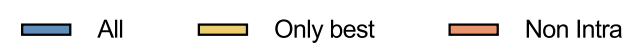

Fig. 4 Evaluation of the effect of different approaches to scoring ambiguous cross-links as Inter-subunit on docking precision. a Comparison of the precision of options All, Only best and Non-Intra. b Comparison of average precision when using SASDs over EUCS for all evaluated scoring functions. Results obtained with SASDs are presented as dotted bars. Values represent the mean precision of all structures in the dataset with SEM. Quade ranking test, followed by Quade's post-hoc test for unreplicated blocked data with Bonferroni correction * p-values $<0.05, * * 0.01, * * 0.001, * * * 0.0001$ 
scoring function (Fig. 4a) and using SASDs outperforms EUCs (Fig. 4b).

\section{Imposing symmetry improves modeling performance}

It has been already reported that cross-linking data can be used to predict if a protein complex is symmetric or not, based on the identified interaction interfaces [7]. We investigated the effect of imposing symmetry to spatial restraints in the scoring function on modeling performance.

In a perfectly symmetric homo-oligomer - most homo-oligomers are symmetric - the distance between residue pair A-B is identical to its counterpart B-A. Distribution of distance differences of these pairs in our homo-dimer dataset shows that this is indeed the case. More than 95\% of EUCs differences are below $1 \AA$ (Additional file 1: Figure S2). Differences of SASDs are a bit more broadly distributed, but still smaller than 5 $\AA$ in more than 90\% (Additional file 1: Figure S3).

Two ways of imposing symmetry were considered:

- Scoring Inter-subunit alternative as matched only if both Inter-subunit distances were matched (Symmetry-matched) and

- Scoring Inter-subunit alternative as matched if at least one Inter-subunit distance is matched and the difference between Inter-subunit distances is smaller than $5 \AA$. (Symmetry-difference).
These two scoring functions were compared to Normal-oriented and Normal-stringent for all three options discussed above (All, Only best, Non-Intra).

Both symmetry imposing scoring functions performed better than both Normal scoring functions in all cases (Fig. 5, Additional file 1: Table S1). Conversely, the differences between Normal-oriented, Symmetry-matched and Symmetry-difference were not statistically significant.

We believe the reason for this comparable performance of Normal-oriented scoring function is that considering cross-links as oriented and scoring both alternatives separately, doubles the score for each cross-link, when both Inter-subunit alternatives are matched. This also favors symmetric models and thus gives comparable results to Symmetry-matched and Symmetry-difference scoring functions, which favorize symmetry by design. However, it needs to be noted that both Symmetry scoring functions evaluate the symmetry by comparing the distances of both Intersubunit alternatives, while Normal-oriented scoring function gains its increase in performance by over scoring the experimental data.

\section{Summary: using SASDs with stringent conditions and imposing symmetry gives the best results}

Based on our comparisons we conclude that choice of method for inter-residue distance calculation, a decision on when to score ambiguous cross-links as Inter-subunit

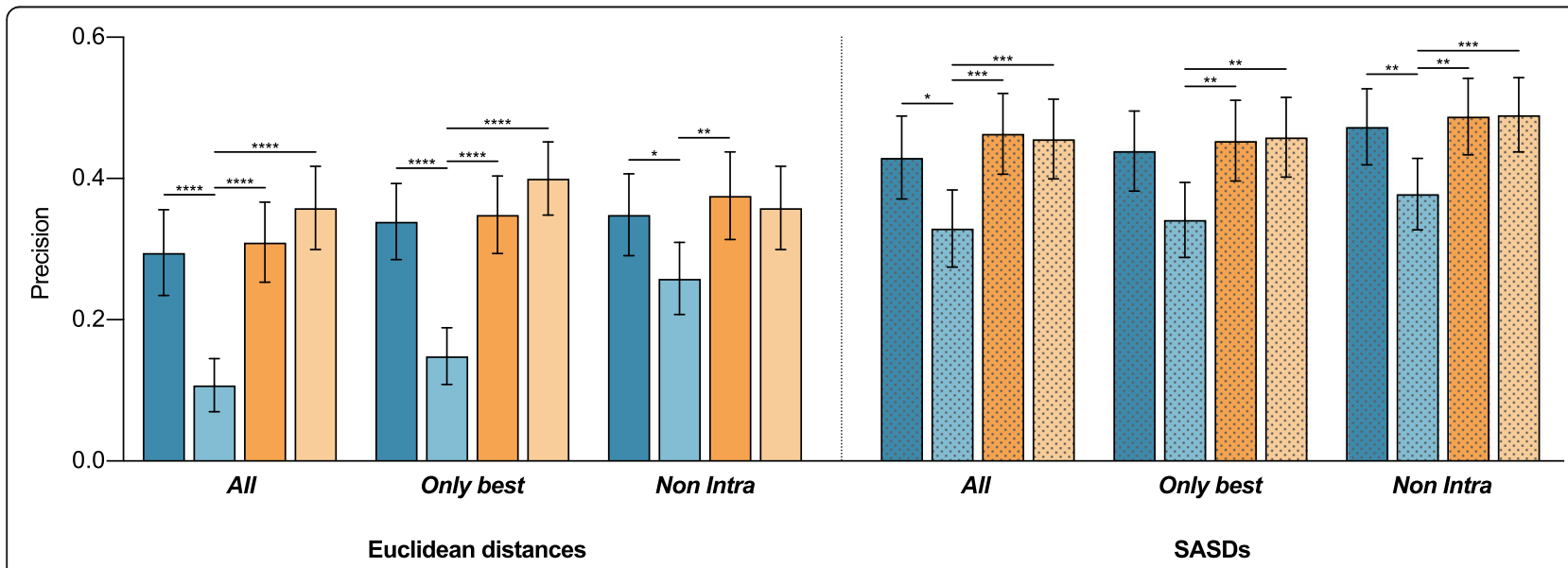

Legend

No symmetry

Imposed symmetry

$\square$ Normal-oriented $\square$ Symmetry-matched

ए Normal-stringent $\rightleftarrows$ Symmetry-difference

Fig. 5 Evaluation of the effect of imposing symmetry on docking precision. Comparison of docking precision with or without directly imposed symmetry. Results obtained with SASDs are presented as dotted bars. Values represent the mean precision of all structures in the dataset with SEM. Quade ranking test, followed by Quade's post-hoc test for unreplicated blocked data with Bonferroni correction ${ }^{*} p$-values <0.05, ** 0.01, *** 0.001, **** 0.0001 
and implying the symmetry in the scoring function itself has an important effect on docking performance.

With regards to the method of inter-residue distance calculation, our analyses clearly show SASDs should be used instead of EUCs.

Ambiguous cross-links should be considered as such as seen from a comparison of Normal and Oblivious scoring functions. Inter-subunit alternatives should be scored only when Intra-subunit alternative is non-accessible - option Non-Intra has the highest precision of the three options analyzed (All, Only Best, Non-Intra).

Including symmetry also improves docking precision, whether symmetry is imposed in the scoring function itself by comparing the distances of Inter-subunit alternatives or favorized as a consequence of improperly addressing cross-linking as oriented and scoring both Inter-subunit alternatives separately.

\section{Discussion}

Many proteins self-assemble to form homo-oligomers, comprised of two or more identical subunits. Structural characterization of homo-oligomeric protein complexes is crucial for the understanding of their role in biological processes. While homo-oligomers have some inherent characteristics such as symmetry, which can be exploited in the process of structure determination, the fact that they are comprised of identical building blocks also presents some additional challenges. In case of modeling protein complexes based on spatial restraints obtained from chemical cross-linking, the obtained linkages cannot be unambiguously assigned as intra- or inter-subunit without labeling of subunits with heavy isotopes, which is not applicable to all investigations [12, 13]. Here we present a comprehensive evaluation of different methods for inter-residue distance calculation and different scoring approaches for resolving this ambiguity based on data analysis alone.

Our results show that using the appropriate methods for inter-residue distance calculations, calculating SASDs instead of EUCs, substantially reduces the number of ambiguous assignations (Fig. 2a), while providing more inter-subunit assignations. Calculating SASDs instead of EUCs generally has a larger effect on Intra-subunit than on Inter-subunit distances (Fig. 2c-d), probably because a direct path (EUC) between two residues on the same subunit is more likely to cross the protein occupied space than when residues are located on different subunits. As distances that cross the protein occupied space cannot represent the actual connection between the residues via the cross-linker, SASDs therefore also produce spatial restraints that are representing the actual experimental information more adequately. The benefit of using SASDs is also evident from our evaluation of how different scoring approaches effect protein-protein docking precision, as scoring functions employing SASDs consistently perform better than those employing EUCs (Figs. 2, 3 and 4). The superiority of SASDs is in agreement with previous evaluations of methods for distance calculations in the modeling of proteins and protein complexes, comprised of different subunits [18].

Even though SASDs can reduce the extent of ambiguity, cross-links that cannot be assigned unambiguously remain. In the literature, there are several different approaches to consider these ambiguous assignations as inter-subunit spatial restraints when modeling homooligomeric protein complexes. The most conservative approach is only to consider those, which can be unambiguously assigned based on overlapping peptide sequences while discarding the rest [21, 22]. Other approaches include assignation of cross-links based on the band of origin in SDS-PAGE analysis [23, 24], considering only those that can't be matched without considering additional subunit $[25,26]$, or assigning crosslinks to the alternative, which has a more favorable distance $[8,27]$. As the first two approaches are based on experimental information additional to the identified connected residues, we focused our evaluation of scoring functions based only on distance information. Results of our comparison reveal that the most conservative option of scoring cross-links as inter-subunit only, when the intra-subunit alternative cannot be matched, gives the best results (Fig. 4a). Even though other options provide a higher total number of restraints, the fact that some of them are falsely assigned as inter-subunit seems to be detrimental for the success of homo-oligomer modeling. While our comparisons were made exclusively on dimers, we believe the underlying principles of our findings can be applied to higher-order homo-oligomers, where several inter-subunit alternatives exist.

Similarly to the findings of others $[7,28]$, we showed that symmetry could be exploited to improve modeling results (Fig. 5). Both of our symmetry imposing scoring functions Symmetry-matched and Symmetry-difference score inter-subunit alternative only when both inter-subunit alternatives (A-B and B-A) are sufficiently similar. The underlying reasoning is related to the recently proposed contact-base symmetry score (CBS) [28], except that CBS evaluates symmetry on the level of the whole protein complex, while we only consider it on the level of individual cross-links. It is surprising, however, that similar precision can be obtained by incorrectly addressing the cross-links as oriented, thus scoring each intersubunit alternative separately.

Further evaluation would be required to determine if this would also hold in a real experiment when not all cross-links are detected, and cross-links that do not correspond to the actual structure (false positives) are also present in the dataset. Unfortunately, we were not able 
to find enough experimental cross-links dataset of C2symmetric homo-dimers with known 3D structures. We compared our findings obtained from simulated crosslinks with four experimental datasets (PDB: 1F05, 1IRI, 2PSN, 4MZV) and with a bootstrapped dataset with only 1, 5, 10 and 20\% coverage (Additional file 1: Figure S4S7). While both results from the experimental dataset as well as results from the simulated lower coverage seem to generally agree with our conclusions based on simulated cross-links, the number of evaluated protein complexes is far too small to enable any reliable evaluation.

\section{Conclusions}

We showed that proper evaluation of chemical crosslinking-based spatial restraints in the modeling of homooligomeric protein complexes can improve the precision of modeling prediction. Modeling algorithms, which employ chemical cross-linking as a type of spatial restraints, should at a minimum address the ambiguity of intra- vs. inter-subunit assignation, when models are scored, instead of addressing each alternative individually. Such optimizations will facilitate investigations of homo-oligomeric protein complexes and in turn, increase our understanding of their structure and function.

\section{Methods}

\section{Comparison of methods for inter-residue distance calculations}

3D structures of homo-oligomers were downloaded from 3D Complex database [29] (version 6, based on PDB database on March 1st, 2015). We included only the structures with at most $90 \%$ amino acid sequence similarity (QS90), high resolution ( $2.5 \AA$ or lower) and at least two subunits, to exclude monomers. Structures, which were found to have incorrect stoichiometry (annotations "YES" and "PROBYES" in PiQSi database [30]) were discarded. During our analysis, we also excluded proteins with multiple structures with different oligomeric states, structures with split polypeptide chains and structures, for which inter-residue distances could not be calculated within three days. This yielded a final dataset of 13,110 structures.

EUCs and SASDs between lysine residues were calculated with Jwalk [18] ( $\mathrm{v}$ 1.3). EUC is calculated as the distance of a straight line between their $C_{\alpha}$-atoms, without considering their solvent accessibility. Jwalk calculates SASD by placing the proteins on a grid, calculating the solvent accessible surface and using a Breadth-First Search to elucidate the shortest distance.

In its original version, Jwalk does not address the intra- vs. inter-subunit ambiguity as each inter-residue connection is defined with residue number and chain id. For our research, we modified the algorithm to treat all connections between the same residue numbers as a group of connections with all available combinations of chain ids. To avoid calculating redundant distances due to symmetry, only distances between lysine pairs, which had at least one endpoint residue located within the first polypeptide chain in the structure were calculated. If there were more Inter-subunit alternatives, only the shortest one was saved. For comparison of assignations, only residue pairs, which had intra- or/and inter-subunit alternative matched (EUC equal or less than the threshold of $33 \AA$ ) and had both intra- and inter-alternative calculated, were considered (1,151,199 residue pairs). These potential cross-links were then assigned as intrasubunit (Intra), inter-subunit (Inter), ambiguous (Ambiguous) or non-matched (Non-accessible) based on the intra- and inter-subunit distances. Briefly, a cross-link was assigned as Intra or Inter if only one of those alternatives had distance $\leq 33 \AA$, Ambiguous if both distances were $\leq 33 \AA$ and Non-accessible if none of the distances was $\leq 33 \AA$ (or endpoint residues were not solvent accessible in case of SASDs).

Figures were prepared with Matplotlib graphics package [31] and Seaborn visualization package [20].

\section{Scoring function comparison dataset}

The experimental cross-linking data of homo-oligomers is scarce. To ensure the results of our scoring function comparisons are generally applicable, we used simulated data. First, we extracted models from the 3D Complex database [29] that matched the following criteria: at most 30\% amino acid sequence similarity (QS30), high resolution ( $2.5 \AA$ or lower), structure is a C2 symmetric dimer and stoichiometry was found correct during manual inspections (annotations "NO" in PiQSi database [30]). Second, proteins with multiple structures with different oligomeric states, structures with split polypeptide chains and structures without any simulated cross-links were removed, yielding the dataset of 47 protein structures. Six protein structures which had less than ten models with $\mathrm{C}_{\alpha}$-RMSD from the recreated initial structure $\leq 10 \AA$ (see below) were excluded from the final dataset. The final dataset thus had 41 protein structures.

\section{Generation of models and calculation of inter-residue distances}

For each protein structure in the dataset, we generated C2-Symmetric and random homo-dimer models using SymmDock [32, 33] and PatchDock [33, 34], respectively. One thousand models with the highest SOAP score [35] from each algorithm were extracted for analysis. In some cases, when PatchDock produced less than 1000 models, all generated models were taken.

Initial structures were also recreated by superimposing the second subunit with the copy of the first one, to ensure the initial structure, had exactly the same chains as 
models. The recreated initial structures were then used to simulate the cross-linking data. We calculated SASDs between all lysine residues using Jwalk [18] (v 1.3) and extracted a list of residue pairs with intra- or inter-subunit SASD equal or below the threshold ( $33 \AA$ ). These residue pairs were considered as simulated cross-linking data, and their EUCs and SASDs were calculated in all models using Jwalk [18] (v 1.3).

\section{Scoring functions}

We used Matched and Non-accessible cross-link (MNXL) [18] scoring function as the basis:

$$
\begin{aligned}
& \text { MNXL score }[\text { distance }] \\
& \quad=\left\{\begin{array}{c}
N(18.62,35.94) \\
-0.1
\end{array} \text { if distance } \leq 33 \AA\right. \text { else }
\end{aligned}
$$

The MNXL score assigns each cross-link a positive value if the distance is under $33 \AA$ (matched). The exact value is calculated based on the probability given by normal distribution with mean (18.62) and variance (35.94) calculated from experimental cross-link database [18]. If the distance is greater than $33 \AA$ - or if any of the endpoint residues is not solvent accessible in case of SASDs - a penalty of -0.1 is assigned (non-accessible).

To enable comparison, the same parameters were used for EUCs, SASDs, intra-subunit, and inter-subunit distances. Models were ranked by total MNXL score (sum of MNXL scores of inter-subunit cross-links). We created several scoring functions that had different criteria when to include a single inter-subunit MNXL score in the inter-subunit MNXL score:

Oblivious: inter-subunit MNXL score is included regardless of the value of its intra-subunit alternative. Normal: if inter-subunit MNXL score is non-accessible, the penalty is not assigned if the intra-subunit alternative is matched but rather scored with a neutral value of 0.0 .

Oriented: both inter-subunit alternatives (A-B and B-A) are scored separately.

Stringent: only the inter-subunit alternative with the higher MNXL score included.

All: inter-subunit MNXL score is included even if the intra-subunit alternative had a higher MNXL score. Only best: inter-subunit MNXL score is included only if it is higher than if the intra-subunit alternative. Non-intra: inter-subunit MNXL score is included only if the intra-subunit alternative is non-accessible. Symmetry matched: inter-subunit MNXL score is included only if both inter-subunit alternatives are matched.

Symmetry-difference: inter-subunit MNXL score is included if at least one of inter-subunit alternatives is matched and if the difference in their distances is smaller than $5 \AA$.

The performance of scoring functions was compared in terms of precision, calculated as the percentage of near-native models in top-10 models with highest total inter-subunit MNXL. A model was considered a nearnative if the $C_{\alpha}$-RMSD from the recreated initial structure was $\leq 10 \AA$.

\section{Comparison of simulated cross-links with experimental data}

We used experimental cross-linking data for four C2symmetric homo-dimers that had high-resolution 3D structures available (PDB: 1F05, 1IRI, 2PSN, 4MZV) [8, $36,37]$. For these four protein complexes, models were generated, scored, and evaluated in the same manner as described above for the scoring function comparison dataset. For comparison, the analysis was also performed with all possible cross-links, defined as in the scoring function comparison dataset analysis and with simulated $1,5,10$ and $20 \%$ coverage (number of obtained crosslinks vs. number of all possible cross-links).

To ensure simulated coverage was representative, 1000 random datasets were bootstrapped for each percentage value [18].

\section{Statistics}

Results of scoring function performance are represented as mean precision \pm SEM. Graphs were drawn with GraphPad Prism version 8.0.0 for Mac (GraphPad Software, San Diego, California USA). Statistical comparison was made with Quade ranking test [38] (STAC python library [39]) followed by Quade's post-hoc test for unreplicated blocked data with Bonferroni correction [38, 40, 41] (scikit-posthocs, v 0.4.0, https://pypi.org/project/scikit-posthocs/).

\section{Additional files}

Additional file 1: Figure S1. Inter-residue distance distributions an Intraand Inter-subunit EUCs distributions. b Distribution distance increases when using SASDs over EUCs for Intra- and Inter-subunit alternatives. Median values were calculated from kernel density estimates [20]. Figure S2. a-c Effect of threshold distance on assignation when using EUCs. The total values obtained with each threshold are shown in header row and column, while the body of the heatmap shows the proportions of combinations. Values in parentheses represent the relative proportion of assignations obtained using a lower threshold, excluding Non-accessible. In row headers, Amb. stands for Ambiguous and Non-acc. Stands for Non-accessible. Figure S3. Distribution of distance differences between both Inter-subunit alternatives in recreated initial structures. Figure S4. Comparison of simulated cross-links with experimental data (PDB: 1F05). For each scoring function, the precision obtained with experimental crosslinks, all possible cross-links and average precision obtained from bootstrapped dataset at 1, 5, 10, and 20\%. a Comparison of Oblivious and Normal scoring functions. b Comparison of scoring options All, Only best and Non-Intra applied to Normal-oriented and Normal-stringent scoring functions. c Comparison of 
symmetry imposing scoring functions Symmetr-matched and Symmetrydifference with Normal scoring functions. Figure S5. Comparison of simulated cross-links with experimental data (PDB: 1IRI). For each scoring function, the precision obtained with experimental cross-links, all possible cross-links and average precision obtained from bootstrapped dataset at 1, 5, 10, and 20\%. a Comparison of Oblivious and Normal scoring functions. b Comparison of scoring options All, Only best and Non-Intra applied to Normal-oriented and Normalstringent scoring functions. c Comparison of symmetry imposing scoring functions Symmetry-matched and Symmetry-difference with Normal scoring functions. Figure S6. Comparison of simulated cross-links with experimental data (PDB: 2PSN). For each scoring function, the precision obtained with experimental cross-links, all possible cross-links and average precision obtained from bootstrapped dataset at 1,5,10, and 20\%. a Comparison of Oblivious and Normal scoring functions. b Comparison of scoring options All, Only best and Non-Intra applied to Normal-oriented and Normal-stringent scoring functions. c Comparison of symmetry imposing scoring functions Symmetry-matched and Symmetry-difference with Normal scoring functions. Figure S7. Comparison of simulated cross-links with experimental data (PDB: 4MZV). For each scoring function, the precision obtained with experimental cross-links, all possible crosslinks and average precision obtained from bootstrapped dataset at 1,5, 10, and 20\%. a Comparison of Oblivious and Normal scoring functions. b Comparison of scoring options All, Only best and Non-Intra applied to Normal-oriented and Normal-stringent scoring functions. c Comparison of symmetry imposing scoring functions Symmetry-matched and Symmetry-difference with Normal scoring functions. Table S1. Comparison of average precisions of scoring functions Normal-oriented, Normal-stringent, Symmetry-matched, and Symmetry-difference. (PDF $366 \mathrm{~kb}$ )

Additional file 2: Table S2. Results of Scoring function comparisons. Results are presented as the precision of each scoring function for homooligomeric protein complex in the analyzed dataset. Precision of both PathDock an Symmdock withouth cross-linking-based distance restrains is also presented. (XLSX $16 \mathrm{~kb})$

\section{Abbreviations}

EUC: Euclidean distance; MNXL: Matched and Non-accessible cross-link; SASD: Solvent accessible surface distance; XL-MS: chemical cross-linking coupled with mass spectrometry

\section{Acknowledgements}

Brigita Lenarčič is gratefully acknowledged for helpful discussions. We also thank Jurij Reščič for access to computer cluster used for calculations.

\section{Authors' contributions}

A. G. and M. P. both conceived the project, performed computations, analyzed the results and wrote the manuscript. G. G. performed a comparison between simulated cross-links and experimental data. All authors read and approved the final manuscript.

\section{Funding}

This research was funded by the Slovenian Research Agency (grants J17119).

\section{Availability of data and materials}

The dataset used and/or analyzed in this study are available from the corresponding author upon reasonable request.

\section{Ethics approval and consent to participate}

Not applicable.

\section{Consent for publication}

Not applicable.

\section{Competing interests}

The authors declare that they have no competing interests.
Received: 18 April 2019 Accepted: 16 August 2019

Published online: 09 September 2019

\section{References}

1. Ward AB, Sali A, Wilson IA. Integrative structural biology. Science. 2013;339: 913-5. https://doi.org/10.1126/science.1228565.

2. Schneidman-Duhovny D, Rossi A, Avila-Sakar A, Kim SJ, Velázquez-Muriel J, Strop $P$, et al. A method for integrative structure determination of proteinprotein complexes. Bioinformatics. 2012;28:3282-9.

3. Levy ED, Teichmann SA. Structural, Evolutionary, and Assembly Principles of Protein Oligomerization. In: Progress in Molecular Biology and Translational Science. 1st edition. Elsevier Inc.; 2013. p. 25-51. doi:https://doi.org/10.1016/ B978-0-12-386931-9.00002-7.

4. Walzthoeni $T$, Joachimiak $L A$, Rosenberger $G$, Röst $H L$, Malmström $L$, Leitner A, et al. xTract: software for characterizing conformational changes of protein complexes by quantitative cross-linking mass spectrometry. Nat Methods. 2015;12:1185-90. https://doi.org/10.1038/ nmeth.3631.

5. Hussain S, Kinnebrew M, Schonenbach NS, Aye E, Han S. Functional consequences of the oligomeric assembly of Proteorhodopsin. J Mol Biol. 2015;427:1278-90. https://doi.org/10.1016/j.jmb.2015.01.004

6. Kahraman A, Herzog F, Leitner A, Rosenberger G, Aebersold R, Malmström L. Cross-link guided molecular modeling with ROSETTA. PLOS One. 2013;8: e73411. https://doi.org/10.1371/journal.pone.0073411.

7. Vreven T, Schweppe DK, Chavez JD, Weisbrod CR, Shibata S, Zheng C, et al. Integrating cross-linking experiments with ab initio protein-protein docking. J Mol Biol. 2018;430:1814-28. https://doi.org/10.1016/j.jmb.2018.04.010.

8. Bullock JMA, Sen N, Thalassinos K, Topf M. Modeling Protein Complexes Using Restraints from Crosslinking Mass Spectrometry. Structure. 2018;:1-10. doi:https://doi.org/10.1016/j.str.2018.04.016.

9. Merkley ED, Rysavy S, Kahraman A, Hafen RP, Daggett V, Adkins JN. Distance restraints from crosslinking mass spectrometry: mining a molecular dynamics simulation database to evaluate lysine-lysine distances. Protein Sci. 2014;23:747-59. https://doi.org/10.1002/pro.2458.

10. Taverner T, Hall NE, O'Hair RAJ, Simpson RJ. Characterization of an antagonist interleukin-6 dimer by stable isotope labeling, cross-linking, and mass spectrometry. J Biol Chem. 2002;277:46487-92.

11. Pettelkau J, Thondorf I, Theisgen S, Lilie H. Schr??der T, Arlt C, et al. structural analysis of guanylyl cyclase-activating protein-2 (GCAP-2) homodimer by stable isotope-labeling, chemical cross-linking, and mass spectrometry. J Am Soc Mass Spectrom. 2013;24:1969-79.

12. Merkley ED, Baker ES, Crowell KL, Orton DJ, Taverner T, Ansong C, et al. Mixed-isotope labeling with LC-IMS-MS for characterization of proteinprotein interactions by chemical cross-linking. J Am Soc Mass Spectrom. 2013;24:444-9. https://doi.org/10.1007/s13361-012-0565-x.

13. Lima DB, Melchior JT, Morris J, Barbosa VC, Chamot-Rooke J, Fioramonte M, et al. Characterization of homodimer interfaces with cross-linking mass spectrometry and isotopically labeled proteins. Nat Protoc. 2018;13:431-58. https://doi.org/10.1038/nprot.2017.113.

14. Schmidt C, Robinson CV. A comparative cross-linking strategy to probe conformational changes in protein complexes. Nat Protoc. 2014;9:2224-36. https://doi.org/10.1038/nprot.2014.144.

15. Morgner N, Schmidt C, Beilsten-Edmands V, Ebong I, Patel NA, Clerico EM, et al. Hsp70 forms antiparallel dimers stabilized by post-translational modifications to position clients for transfer to Hsp90. Cell Rep. 2015;11: 759-69. https://doi.org/10.1016/j.celrep.2015.03.063.

16. Schmidt C, Beilsten-Edmands V, Robinson C V. The joining of the Hsp90 and Hsp70 chaperone cycles yields transient interactions and stable intermediates: insights from mass spectrometry. Oncotarget. 2015;6:484-93. doi:https://doi.org/10.18632/oncotarget.4954.

17. Kahraman A, Malmström L, Aebersold R. Xwalk: computing and visualizing distances in cross-linking experiments. Bioinformatics. 2011;27:2163-4. https://doi.org/10.1093/bioinformatics/btr348.

18. Bullock JMA, Schwab J, Thalassinos K, Topf M. The importance of nonaccessible crosslinks and solvent accessible surface distance in modelling proteins with restraints from crosslinking mass spectrometry. Mol Cell Proteomics. 2016::12-5. doi:https://doi.org/10.1074/mcp. M116.058560.

19. Degiacomi MT, Schmidt C, Baldwin AJ, Benesch JLP. Accommodating Protein Dynamics in the Modeling of Chemical Crosslinks. Structure. 2017; 25:1751-1757.e5. doi:https://doi.org/10.1016/j.str.2017.08.015. 
20. Waskom M, Botvinnik O, O'Kane D, Hobson P, Lukauskas S, Gemperline DC et al. mwaskom/seaborn: v0.8.1 (September 2017). 2017. doi:https://doi. org/10.5281/ZENODO.883859.

21. Haupt C, Hofmann T, Wittig S, Kostmann S, Politis A, Schmidt C. Combining Chemical Cross-linking and Mass Spectrometry of Intact Protein Complexes to Study the Architecture of Multi-subunit Protein Assemblies. J Vis Exp. 2017::1-12. doi:https://doi.org/10.3791/56747.

22. Wittig S, Haupt C, Hoffmann W, Kostmann S, Pagel K, Schmidt C. Oligomerisation of Synaptobrevin-2 studied by native mass spectrometry and chemical cross-linking. J Am Soc Mass Spectrom. 2019;30:149-60. https://doi.org/10.1007/s13361-018-2000-4.

23. Bennett KL, Kussmann M, Mikkelsen M, Roepstorff P, Björk P, Godzwon M, et al. Chemical cross-linking with thiol-cleavable reagents combined with differential mass spectrometric peptide mapping-a novel approach to assess intermolecular protein contacts. Protein Sci. 2000;9:1503-18. https:// doi.org/10.1110/ps.9.8.1503

24. Liu Z, Szarecka A, Yonkunas M, Speranskiy K, Kurnikova M, Cascio M. Crosslinking constraints and computational models as complementary tools in modeling the extracellular domain of the Glycine receptor. PLoS One. 2014;9:e102571. https://doi.org/10.1371/journal.pone.0102571.

25. Hall Z, Schmidt C, Politis A. Uncovering the early assembly mechanism for Amyloidogenic $\beta 2$-microglobulin using cross-linking and native mass spectrometry. J Biol Chem. 2016;291:4626-37. https://doi.org/10.1074/jbc. M115.691063.

26. Gaber A, Kim SJ, Kaake RM, Benčina M, Krogan N, Šali A, et al. EpCAM homo-oligomerization is not the basis for its role in cell-cell adhesion. Sci Rep. 2018;8:13269. https://doi.org/10.1038/s41598-018-31482-7.

27. LoPiccolo J, Kim SJ, Shi Y, Wu B, Wu H, Chait BT, et al. Assembly and molecular architecture of the phosphoinositide 3-kinase p85a homodimer. J Biol Chem. 2015;290:30390-405. https://doi.org/10.1074/ jbc.M115.689604.

28. Maheshwari S, Brylinski M. Predicted binding site information improves model ranking in protein docking using experimental and computergenerated target structures. BMC Struct Biol. 2015;15:23. https://doi.org/1 0.1186/s12900-015-0050-4.

29. Levy ED, Pereira-Leal JB, Chothia C, Teichmann SA. 3D complex: a structural classification of protein complexes. PLoS Comput Biol. 2006;2:e155. https:// doi.org/10.1371/journal.pcbi.0020155.

30. Levy ED. PiQSi: protein quaternary structure investigation. Structure. 2007;15: 1364-7. https://doi.org/10.1016/j.str.2007.09.019.

31. Hunter JD. Matplotlib: a 2D graphics environment. Comput Sci Eng. 2007;9: 90-5. https://doi.org/10.1109/MCSE.2007.55.

32. Schneidman-Duhovny D, Inbar Y, Nussinov R, Wolfson HJ. Geometry-based flexible and symmetric protein docking. Proteins Struct Funct Bioinforma. 2005;60:224-31. https://doi.org/10.1002/prot.20562.

33. Schneidman-Duhovny D, Inbar $Y$, Nussinov R, Wolfson HJ. PatchDock and SymmDock: servers for rigid and symmetric docking. Nucleic Acids Res. 2005;33 Web Server:W363-7. doi:https://doi.org/10.1093/ nar/gki481.

34. Duhovny D, Nussinov R, Wolfson HJ. Efficient unbound docking of rigid molecules. In: Algorithms in Bioinformatics; 2002. p. 185-200. https://doi. org/10.1007/3-540-45784-4_14.

35. Dong GQ, Fan H, Schneidman-Duhovny D, Webb B, Sali A. Optimized atomic statistical potentials: assessment of protein interfaces and loops. Bioinformatics. 2013;29:3158-66. https://doi.org/10.1093/bioinformatics/ btt560.

36. Chavez JD, Schweppe DK, Eng JK, Bruce JE. In vivo conformational dynamics of Hsp90 and its interactors. Cell Chem Biol. 2016;23:716-26. https://doi.org/10.1016/j.chembiol.2016.05.012.

37. Gaber A, Kim SJ, Kaake RM, Benčina M, Krogan N, Šali A, et al. EpCAM homo-oligomerization is not the basis for its role in cell-cell adhesion. Sci Rep. 2018;8

38. Quade D. Using weighted rankings in the analysis of complete blocks with additive block effects. J Am Stat Assoc. 1979;74:680. https://doi.org/10.23 07/2286991

39. Rodriguez-Fdez I, Canosa A, Mucientes M, Bugarin A. STAC: A web platform for the comparison of algorithms using statistical tests. In: 2015 IEEE International Conference on Fuzzy Systems (FUZZ-IEEE). IEEE; 2015. p. 1-8. doi:https://doi.org/10.1109/FUZZ-IEEE.2015.7337889.
40. Conover WJ. Practical nonparametric statistics, 3rd. Edition: Wiley; 1999.

41. Heckert NA, Filliben JJ. NIST handbook 148: Dataplot reference manual volume 2: let subcommands and library functions. National Institute of Standards and Technology Handbook Series; 2003.

\section{Publisher's Note}

Springer Nature remains neutral with regard to jurisdictional claims in published maps and institutional affiliations.
Ready to submit your research? Choose BMC and benefit from:

- fast, convenient online submission

- thorough peer review by experienced researchers in your field

- rapid publication on acceptance

- support for research data, including large and complex data types

- gold Open Access which fosters wider collaboration and increased citations

- maximum visibility for your research: over $100 \mathrm{M}$ website views per year

At BMC, research is always in progress.

Learn more biomedcentral.com/submissions 